\title{
Inflammatory bowel disease increases the risk of Parkinson's disease: a Danish nationwide cohort study 1977-2014
}

\author{
Marie Villumsen, ${ }^{1}$ Susana Aznar, ${ }^{2}$ Bente Pakkenberg, ${ }^{2,3}$ Tine Jess, $^{1}$ Tomasz Brudek ${ }^{2}$
}

${ }^{1}$ Center for Clinical Research and Disease Prevention Bispebjerg and Frederiksberg Hospital, Copenhagen, Denmark ${ }^{2}$ Research Laboratory for Stereology and Neuroscience, Bispebjerg and Frederiksberg Hospital, Copenhagen, Denmark ${ }^{3}$ Faculty of Health, Institute of Clinical Medicine, University of Copenhagen, Copenhagen, Denmark

Correspondence to Dr Tomasz Brudek, Research Laboratory for Stereology and Neuroscience, Bispebjerg and Frederiksberg Hospital, Copenhagen DK-2400, Denmark; tomasz.brudek@ regionh.dk

Received 13 November 2017 Revised 21 March 2018 Accepted 29 March 2018 Published Online First 21 May 2018

\section{Linked}

- http://dx.doi.org/10.1136/ gutjnl-2018-316537

- http://dx.doi.org/10.1136/ gutjnl-2018-316937

Check for updates

To cite: Villumsen $M$, Aznar S, Pakkenberg B, et al. Gut 2019;68:18-24.

\section{ABSTRACT \\ Objective Intestinal inflammation has been} suggested to play a role in development of Parkinson's disease (PD) and multiple system atrophy (MSA). To test the hypothesis that IBD is associated with risk of PD and MSA, we performed a nationwide populationbased cohort study.

Design The cohort consisted of all individuals diagnosed with IBD in Denmark during 1977-2014 ( $n=76477$ ) and non-IBD individuals from the general population, who were comparable in terms of gender, age and vital status ( $n=7548$ 259). All cohort members were followed from IBD diagnosis/index date to occurrence of PD and MSA (according to the Danish National Patient Register).

Results Patients with IBD had a $22 \%$ increased risk of PD as compared with non-IBD individuals ( $H R=1.22$; $95 \% \mathrm{Cl} 1.09$ to 1.35$)$. The increased risk was present independently of age at IBD diagnosis, gender or length of follow-up. The overall incidence of MSA was low in our study, and the regression analysis suggested a tendency towards higher risk of developing MSA in patients with IBD as compared with non-IBD individuals ( $\mathrm{HR}=1.41 ; 95 \% \mathrm{Cl} 0.82$ to 2.44$)$. Estimates were similar for women and men. The increased risk of parkinsonism was significantly higher among patients with UC (HR=1.35; 95\% Cl 1.20 to 1.52) and not significantly different among patients with Crohn's disease ( $H R=1.12 ; 95 \% \mathrm{Cl} 0.89$ to 1.40).

Conclusions This nationwide, unselected, cohort study shows a significant association between IBD and later occurrence of PD, which is consistent with recent basic scientific findings of a potential role of $\mathrm{GI}$ inflammation in development of parkinsonian disorders.

\section{INTRODUCTION}

The IBD, Crohn's disease (CD) and ulcerative colitis (UC) are chronic conditions with onset in young adulthood. In UC, inflammation is superficial and only affects the colon, whereas in CD, inflammation is transmural and may affect the entire GI tract. ${ }^{1}$ IBD is characterised by chronic proinflammatory immune activity, a trait which is now also suggested to be a fundamental element of neurodegenerative disorders. Intestinal inflammation may be of particular relevance in the pathogenesis of parkinsonism, including Parkinson's disease $(\mathrm{PD})^{2}$ and multiple system atrophy (MSA).

\section{Significance of this study}

What is already known on this subject?

- It is proved that Parkinson's disease (PD) is restricted to impairment of movements and it involves several non-motor clinical features including $\mathrm{GI}$ dysfunctions.

- These dysfunctions appear early in the disease and add significantly to PD-related complications.

- Enteric inflammation, the signature symptom of IBD, can also occur in patients with PD and may reflect the earliest manifestations of $P D$ pathology.

What are the new findings?

- In this nationwide population-based study covering the period 1977-2014, we found a significantly increased risk of Parkinson's disease in patients with IBD compared with non-IBD individuals.

- This strengthens the theory of a 'gut-brain axis' where the intestinal environment influences the functioning of the central nervous system and intestinal imbalance may precede PD pathology.

How might it impact on clinical practice in the foreseeable future?

- The study suggests that clinicians should be aware of symptoms of parkinsonism in patients with IBD, and the study demonstrates the need for further investigation into the role of intestinal inflammation and braingut-microbiome axis in the aetiology of parkinsonism.

- The identification of risk factors associated with prodromal phases of PD may allow us for early intervention studies that could modify or slow down disease progress.

Intracellular aggregates of $\alpha$-synuclein ( $\alpha$-syn) are pathological hallmarks of parkinsonism, with $\alpha$-syn being a major constituent of neuronal Lewy bodies in PD, whereas in MSA, $\alpha$-syn aggregates are found mainly in oligodendroglia, ${ }^{34}$ with phosphorylation at serin-129 being the most dominant pathological form (p- $\alpha$-syn). ${ }^{5}$ Of interest, a specific pattern of $p$ - $\alpha$-syn aggregates can be identified in the enteric nervous system (ENS) in patients with 
PD. This accumulation is most likely associated with inflammatory processes and intestinal barrier dysfunctions. ${ }^{6}$

IBD is associated with perturbation in the intestinal immune homeostasis due to failure in the immune response to intestinal microbiota. ${ }^{7}$ It is known that there is a genetic susceptibility for IBD. Genome-wide association studies have reported the leucine-rich repeat kinase 2 (LRRK2) gene to be one of the major gene loci associated with increased susceptibility for $\mathrm{CD} .^{8-10}$ Interestingly, LRRK2 is the gene most commonly associated with both familial and sporadic PD and MSA. ${ }^{10}{ }^{11}$ LRRK2 was recently found to be involved in regulation of inflammatory responses and $\alpha$-syn clearance. ${ }^{12}$ Our own observations suggest that a defect in the innate immune response could be responsible for the $\alpha$-syn accumulation. ${ }^{13}$

Hence, the current state of the art in pathogenic mechanisms behind parkinsonism supports a link between gut inflammation and development of PD and MSA. However, real-life evidence from epidemiological studies is lacking, except for a minor study of $5 \%$ of the Taiwanese population, suggesting that especially CD may be associated with development of PD. ${ }^{14}$

We, therefore, aimed to perform a nationwide and hence unselected cohort study of all patients diagnosed with IBD in Denmark during 1977-2014 to assess the association with later development of the two forms of parkinsonism, idiopathic PD and MSA.

\section{METHODS}

\section{Study population}

Our study was based on all individuals aged 15 years or older and living in Denmark during 1977 and 2014 ( $\mathrm{n}=8858$ 367). In Denmark, all citizens are assigned a unique individual personal identification number (Civil Personal Registration (CPR) number) at birth or immigration by the Danish Civil Registration System. ${ }^{15}$ The CPR number enables linkage to other national registers including the Danish National Patient Register (NPR), a register containing information on all inpatient hospitalisations in somatic wards in Denmark since 1977 and outpatient hospital contacts in emergency rooms and ambulatory settings since 1995 . $^{16}$

In the NPR we identified all patients with a diagnosis of IBD during 1977 to 2014 using primary or secondary diagnosis codes classified with the International Classification of Diseases Eighth or Tenth Revision (ICD-8/ICD-10). The definition of IBD included CD (ICD-8 codes 56300-56 309 and ICD-10 code group K50) and UC (ICD-8 code 56319 or 56904 and ICD-10 code group K51). The validity of the registration with IBD diagnoses in the NPR has previously been estimated to be $97 \%$ for CD and $90 \%$ for UC. ${ }^{17}$ Patients who had a diagnosis of both CD and UC in the NPR were classified based on their last diagnosis but with follow-up starting at time of first registration with one of the diagnoses.

To increase comparability between patients with IBD and non-IBD individuals, we did not compare patients with IBD with all individuals in the background population, but matched each patient with IBD with all comparable individuals without IBD in terms of gender, age (date of birth \pm 1 week) and vital status on the date of first IBD diagnosis. Patients with IBD and non-IBD individuals with no comparable matches were excluded.

We used NPR to identify all individuals in our study population with a diagnosis (primary or secondary) of PD (ICD-8 code 342 and ICD-10 code group G20) or MSA (ICD-10 codes DG232-DG233), ${ }^{18}{ }^{19}$ and to identify comorbidities among cohort members (based on the method described by Christensen $\left.e t a^{20}\right)$. Comorbidity scores were handled as a categorical variable.

Using the Danish Civil Registration System, which also contains continuously updated information on demographics in the Danish population, ${ }^{15}$ we obtained information on disappearance, emigration and death for our study population.

\section{Statistical analyses}

All cohort members were followed from index date (date of IBD diagnosis or comparable date in matches) until PD or MSA, emigration, death or end of the study, 31 December 2014, whichever event occurred first. Persons diagnosed with PD or MSA before the index date were excluded from the analysis. By Cox proportional hazard regression, using time since index date as underlying time scale, we estimated the risk of PD and MSA, respectively, in patients with IBD compared with non-IBD individuals. Results were reported by hazard rate ratios (HR) with 95\% CIs, which were calculated IBD combined and for UC and CD separately. HRs were adjusted for Charlson Comorbidity Index.

In addition to the overall estimate, we estimated gender-specific HRs of PD and MSA. Further stratification of the analysis of MSA was not possible due to the low number of cases. In the analyses of PD, we explored if age at IBD diagnosis influenced the association by stratifying the analysis in three age groups ( $<40$ years, $40-65$ years, $>65$ years). Stratification of the HRs on follow-up time was performed to evaluate if time between IBD influenced the risk of developing PD. To test the robustness of our analyses, we also performed a number of sensitivity analyses. Statistical analyses were performed with SAS V.9.4 (SAS Institute).

\section{Patient involvement}

No patients were involved in setting the research question or the outcome measures, nor were they involved in developing plans for design or implementation of the study. No patients were asked to advise on interpretation or writing up of results. We plan to disseminate the results of the research to the relevant patient community.

\section{RESULTS}

In our nationwide cohort of 8858367 individuals, a total of 76594 patients were diagnosed with IBD at age 15 years or older during 1977-2014. Individuals in the source population who were not comparable with patients diagnosed with IBD in terms of gender and week of birth were excluded $(n=1$ 310 108). The final cohort comprised 76477 patients with IBD and 7548259 non-IBD individuals. Characteristics of patients with IBD and matched non-IBD individuals are shown in table 1. Patients with IBD had more comorbidities than non-IBD individuals $(\mathrm{p}<0.001)$, which was taken into account in the analyses.

During more than 83 million years of follow-up, 335 patients with IBD (0.4\%) and 39784 non-IBD individuals $(0.5 \%)$ were diagnosed with PD, whereas 13 patients with IBD $(0.02 \%)$ and 866 non-IBD individuals $(0.01 \%)$ were diagnosed with MSA (figure 1). A total of 41 patients with IBD were excluded due to PD or MSA prior to IBD diagnosis.

The cumulative probability of PD was significantly higher in patients with IBD than in non-IBD individuals during up to 37 years of follow-up (figure $2, \log$ rank, $\mathrm{p}=0.02$ ). The mean interval from IBD to diagnosis of PD was 11.7 years (range, 
Table 1 Characteristics of the Danish nationwide study cohort (1977-2014)

\begin{tabular}{|c|c|c|c|c|c|c|c|c|}
\hline \multirow[b]{2}{*}{ Characteristics } & \multicolumn{2}{|c|}{ Individuals diagnosed with CD } & \multicolumn{2}{|c|}{ Non-IBD individuals } & \multicolumn{2}{|c|}{ Individuals diagnosed with UC } & \multicolumn{2}{|c|}{ Non-IBD individuals } \\
\hline & $\mathrm{n}$ & $\%$ & $\mathrm{n}$ & $\%$ & $\mathrm{n}$ & $\%$ & $\mathbf{n}$ & $\%$ \\
\hline Total & 24594 & & 2547429 & & 51822 & & 4927119 & \\
\hline \multicolumn{9}{|l|}{ Gender } \\
\hline Female & 14195 & 57.7 & 1299156 & 51.0 & 27410 & 52.9 & 2374342 & 48.2 \\
\hline Male & 10399 & 42.3 & 1248273 & 49.0 & 24357 & 47.1 & 2522777 & 51.8 \\
\hline \multicolumn{9}{|c|}{ Comorbidity index } \\
\hline 0 & 12315 & 50.1 & 1741926 & 68.4 & 25361 & 49.0 & 2948994 & 59.9 \\
\hline $1-2$ & 7188 & 29.2 & 510825 & 20.1 & 14994 & 29.0 & 1197925 & 24.3 \\
\hline $3-4$ & 2860 & 11.6 & 168551 & 6.6 & 6467 & 12.5 & 450197 & 9.1 \\
\hline $5-6$ & 1768 & 7.2 & 106586 & 4.2 & 3921 & 7.6 & 278940 & 5.7 \\
\hline$>7$ & 463 & 1.9 & 19541 & 0.8 & 1024 & 2.0 & 51063 & 1.0 \\
\hline \multicolumn{9}{|c|}{ Age at IBD diagnosis (years)* } \\
\hline$<40$ & 13477 & 54.8 & 1603750 & 62.9 & 22105 & 42.7 & 2369365 & 48.1 \\
\hline $40-65$ & 7095 & 28.8 & 599045 & 23.5 & 18019 & 34.8 & 1500711 & 30.5 \\
\hline$>65$ & 4022 & 16.4 & 344634 & 13.5 & 11643 & 22.5 & 1057043 & 21.5 \\
\hline \multicolumn{9}{|c|}{ Year at IBD diagnosist } \\
\hline 1977-1984 & 2971 & 12.1 & 405671 & 15.9 & 6935 & 13.4 & 1092950 & 22.2 \\
\hline 1985-1994 & 4627 & 18.8 & 482049 & 18.9 & 8672 & 16.8 & 833817 & 16.9 \\
\hline 1995-2005 & 7234 & 29.4 & 653038 & 25.6 & 15635 & 30.2 & 1296167 & 26.3 \\
\hline 2005-2014 & 9762 & 39.7 & 1006671 & 39.5 & 20525 & 39.6 & 1704185 & 34.6 \\
\hline
\end{tabular}

${ }^{*}$ Age at IBD diagnosis is age at matching for non-IBD individuals in the background cohort.

† Number of non-IBD individuals matched with patients with IBD diagnosed in the respective period. All eligible matches are chosen. A match can be matched to more than one patient with IBD.

CD, Crohn's disease; UC, ulcerative colitis.

0.3-37.6 years). Mean age at diagnosis of PD was similar in patients with IBD (73.7 years) and non-IBD individuals (73.8 years) ( $\mathrm{t}$-test; $\mathrm{p}=0.58$ ). This was also the case when analysing CD and UC separately. Using Cox regression, we observed a $24 \%$ increased risk of PD in patients with IBD as compared with non-IBD individuals (HR 1.24; 95\% CI 1.12 to 1.34 ) in crude analysis and a $22 \%$ increased risk (HR 1.22; 95\% CI 1.09 to 1.35 ) after adjustment for comorbidity index (table 2). The increased risk was present both in women (HR 1.23; 95\% CI 1.05 to 1.45 ) and men (HR $1.20 ; 95 \%$ CI 1.04 to 1.39 ), it was similar in patients with IBD diagnosed at age $<40$ years (HR $1.30 ; 95 \%$ CI 1.19 to 1.52 ), 40-65 years (HR $1.25 ; 95 \%$ CI 1.06 to 1.47 ) and $>65$ years (HR 1.22 ; 95\% CI 0.82 to 1.82 ), and it was independent of length of follow-up (table 2).
The overall incidence of MSA was low in our study, and the cumulative probability of MSA was not significantly higher in patients with IBD than in non-IBD individuals (figure 3). Our regression analysis suggested however a tendency towards higher risk of developing MSA in patients with IBD as compared with non-IBD individuals (adjusted HR 1.41; 95\% CI 0.82 to 2.44) (table 3). Estimates were similar for women and men (table 3).

When analysing the composite outcome of PD and MSA (in total 40077 PD cases and 879 MSA cases), patients with IBD had a 29\% higher risk of PD or MSA (adjusted HR 1.29; 95\% CI 1.16 to 1.44 ) than IBD or non-IBD individuals. The increased risk of PD and MSA was slightly higher among patients with UC (adjusted HR 1.35; 95\% CI 1.20 to 1.52 ) than among patients with CD (adjusted HR 1.12; 95\% CI 0.89 to 1.40), but CIs

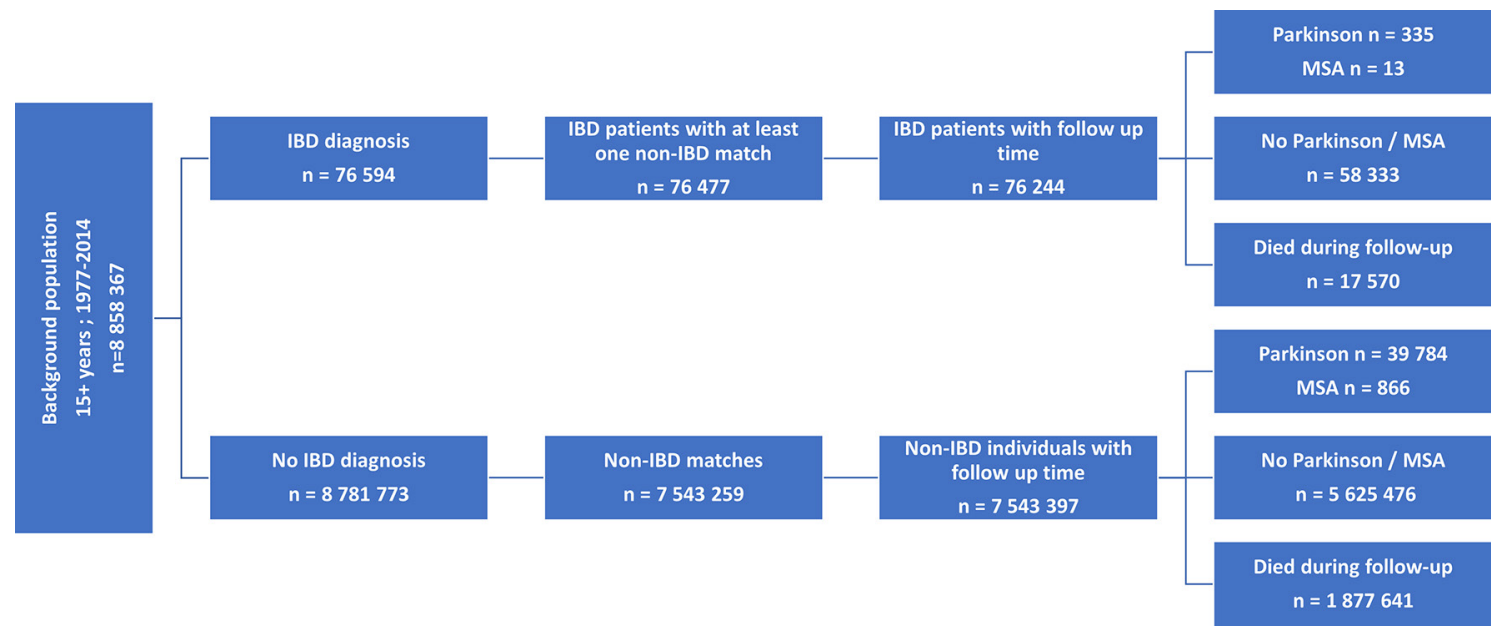

Figure 1 Flow chart of the study population. MSA, multiple system atrophy. 


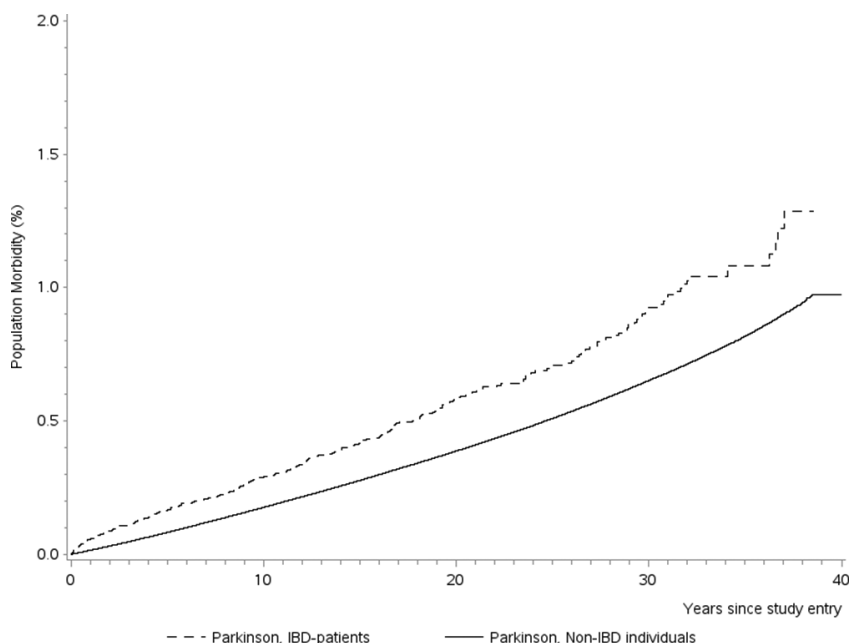

Figure 2 Kaplan-Meier curve illustrating the cumulative incidence comparison of Parkinson's disease for patients diagnosed with (solid line) or without (dashed line) IBD.

were overlapping. Accordingly, patients with UC were found to have a 3\% higher risk of PD and MSA than patients with CD (HR 1.035 ; 95\% CI 1.03 to 1.04 ; $\mathrm{p}<0.001$ ).

\section{Sensitivity analyses}

To test the robustness of our analyses, we did a number of sensitivity analyses. First, to account for the possibility that some patients had PD with initial intestinal symptoms misclassified as IBD, we did a sensitivity analysis excluding the first 2 years of follow-up after first diagnosis with IBD. This did not change the adjusted estimate of parkinsonism in patients with IBD compared with non-IBD individuals markedly (HR 1.14;

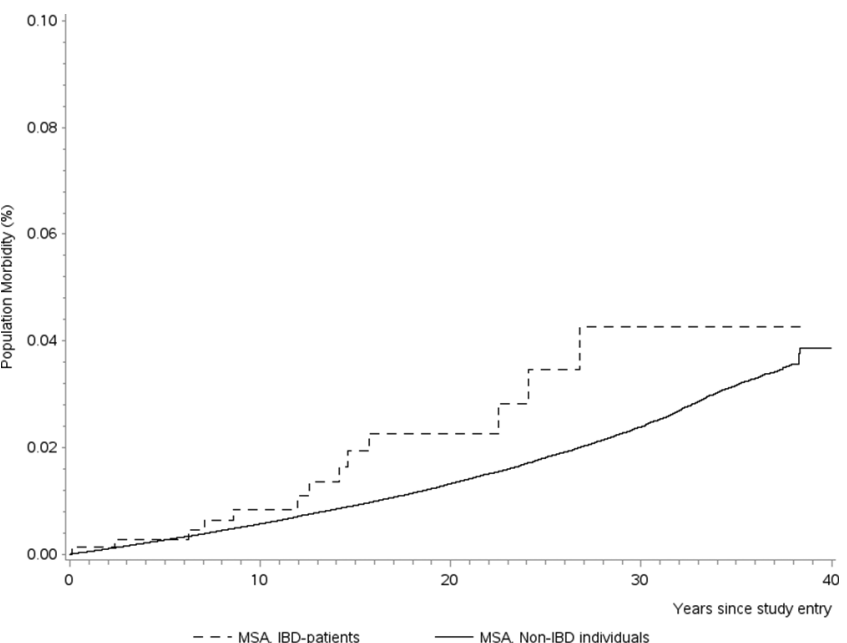

Figure 3 Kaplan-Meier curve illustrating the cumulative incidence comparison of multiple system atrophy (MSA) for patients diagnosed with (solid line) or without (dashed line) IBD.

95\% CI 1.01 to 1.28 ). To confirm the robustness of data according to data availability over calendar time, we also did a sensitivity analysis only including 53329 patients with IBD diagnosed in 1995 or later, where complete information on outpatient visits was available. This did not change the adjusted estimate of parkinsonism in patients with IBD compared with non-IBD individuals markedly either (HR 1.14; 95\% CI 0.97 to 1.34 ). In a further sensitivity analysis, we excluded 2242 patients with IBD (and their matches) registered with only one short (less than a week) inpatient contact with IBD as primary diagnosis. This left us with a subset of patients with IBD with two or more contacts, among whom the adjusted estimate

Table 2 Risk of Parkinson's disease in patients with IBD: a Danish nationwide cohort study 1977-2014

\begin{tabular}{|c|c|c|c|c|c|c|}
\hline & \multicolumn{2}{|c|}{ Patients with IBD } & \multicolumn{2}{|c|}{ Non-IBD individuals } & \multicolumn{2}{|c|}{$\begin{array}{l}\text { HR for individuals diagnosed with IBD compared with non- } \\
\text { IBD individuals (reference) }\end{array}$} \\
\hline & Events & Rate* & Events & Rate* & Crude HR $(95 \% \mathrm{CI})$ & Adjustedt HR (95\% CI) \\
\hline \multicolumn{7}{|l|}{ IBD } \\
\hline All & 335 & 0.37 & 39784 & 0.22 & $1.24(1.12$ to 1.38$)$ & 1.22 (1.09 to 1.35$)$ \\
\hline \multicolumn{7}{|l|}{ Gender } \\
\hline Female & 148 & 0.29 & 17950 & 0.19 & 1.27 (1.08 to 1.49$)$ & $1.23(1.05$ to 1.45$)$ \\
\hline Male & 187 & 0.46 & 21834 & 0.26 & $1.23(1.06$ to 1.41$)$ & $1.20(1.04$ to 1.39$)$ \\
\hline \multicolumn{7}{|c|}{ Charlson Comorbidity Index } \\
\hline 0 & 59 & 0.13 & 8947 & 0.09 & 1 (reference) & \\
\hline $1-2$ & 134 & 0.49 & 17613 & 0.39 & 1.83 (1.81 to 1.84$)$ & \\
\hline $3-4$ & 94 & 0.86 & 8994 & 0.66 & 2.04 (2.02 to 2.06$)$ & \\
\hline $5-6$ & 38 & 0.63 & 3541 & 0.50 & 1.68 (1.66 to 1.71$)$ & \\
\hline$>7$ & 10 & 0.61 & 689 & 0.51 & 1.59 (1.55 to 1.63$)$ & \\
\hline \multicolumn{7}{|c|}{ Age at IBD diagnosis (years) } \\
\hline$<40$ & 24 & 0.05 & 6374 & 0.04 & $1.32(0.88$ to 1.96$)$ & $1.22(0.82$ to 1.82$)$ \\
\hline $40-65$ & 145 & 0.49 & 22282 & 0.36 & 1.32 (1.12 to 1.56$)$ & 1.25 (1.06 to 1.47$)$ \\
\hline$>65$ & 166 & 1.73 & 11128 & 1.36 & 1.33 (1.14 to 1.54$)$ & $1.30(1.12$ to 1.52$)$ \\
\hline \multicolumn{7}{|c|}{ Follow-up time (years) } \\
\hline$<1$ & 43 & 16.41 & 1135 & 5.68 & 1.44 (1.07 to 1.94$)$ & 1.36 (1.01 to 1.84$)$ \\
\hline $1-20$ & 230 & 0.48 & 19891 & 0.31 & 1.24 (1.09 to 1.41$)$ & 1.15 (1.01 to 1.31$)$ \\
\hline$>20$ & 62 & 0.15 & 18758 & 0.10 & 1.37 (1.07 to 1.76$)$ & $1.26(0.98$ to 1.62$)$ \\
\hline
\end{tabular}

HRs are stratified by matching factors (gender and age).

*Incidence rate per 1000 person-years.

tHRs are adjusted for Charlson Comorbidity Index. 
Table 3 Risk of multiple system atrophy in patients with IBD: a Danish nationwide cohort study 1977-2014

\begin{tabular}{|c|c|c|c|c|c|c|}
\hline & \multicolumn{2}{|l|}{ IBD } & \multicolumn{2}{|c|}{ Non-IBD individuals } & \multicolumn{2}{|c|}{$\begin{array}{l}\text { HR for individuals diagnosed with IBD compared with non- } \\
\text { IBD individuals (reference) }\end{array}$} \\
\hline & Events* & Ratet & Events & Rate & Crude HR $(95 \% \mathrm{Cl})$ & Adjusted HR $¥(95 \% \mathrm{Cl})$ \\
\hline \multicolumn{7}{|l|}{ IBD } \\
\hline All & 13 & 0.014 & 866 & 0.008 & 1.63 (0.95 to 2.81$)$ & 1.41 (0.82 to 2.44$)$ \\
\hline \multicolumn{7}{|l|}{ Gender } \\
\hline Female & 6 & 0.014 & 440 & 0.008 & 1.61 (0.77 to 3.38$)$ & 1.41 (0.67 to 2.96$)$ \\
\hline Male & 7 & 0.015 & 426 & 0.007 & 1.60 (0.72 to 3.56$)$ & 1.38 (0.62 to 3.08$)$ \\
\hline \multicolumn{7}{|c|}{ Charlson Comorbidity Index } \\
\hline 0 & $<5$ & - & 237 & - & 1 (reference) & \\
\hline $1-2$ & $<5$ & - & 326 & - & 2.43 (2.32 to 2.54$)$ & \\
\hline $3-4$ & $<5$ & - & 180 & - & 3.46 (3.27 to 3.66$)$ & \\
\hline $5-6$ & $<5$ & - & 95 & - & 3.74 (3.49 to 4.00$)$ & \\
\hline$>7$ & $<5$ & - & 28 & - & 4.16 (3.70 to 4.69$)$ & \\
\hline
\end{tabular}

HRs are stratified by matching factors (genderand age).

*According to the regulation of the Danish Health Data Authority fewer than five events must be anonymised.

tIncidence rate per 1000 person-years.

¥HRs are adjusted for Charlson Comorbidity Index.

of parkinsonism also remained fairly unchanged (HR 1.21; $95 \%$ CI 1.09 to 1.35 ). In our data, many outpatient registrations were long (years) and comprised several visits. Inpatient contacts parallel to outpatient treatment were therefore common. In a sensitivity analysis of 33839 patients with IBD (44.2\%) who had both inpatient and outpatient visits registered, the adjusted estimate of parkinsonism was again fairly unchanged (HR 1.22; 95\% CI 1.04 to 1.43). In patients with IBD, only having inpatient $(22.2 \%)$ or outpatient $(33.6 \%)$ visits registered, the adjusted estimate of parkinsonism in patients with IBD compared with non-IBD individuals was 1.15 (95\% CI 0.95 to 1.39 ) and 1.27 (95\% CI 1.00 to 1.60$)$, respectively.

\section{DISCUSSION}

In this nationwide Danish cohort study of all patients with IBD followed for more than 8 million person-years, we found a significantly increased risk of PD when comparing patients with IBD with non-IBD individuals from the general Danish population. We also observed a tendency towards increased risk of MSA, although power was limited for this disease subtype. The risk of parkinsonism seemed independent of age at IBD diagnosis and of gender, whereas patients with UC had slightly higher risk than patients with CD.

To our knowledge, this is the first epidemiological study investigating the risk of parkinsonism in an unselected, nationwide cohort of patients with IBD with long-term follow-up from a country with easy and free access to healthcare. The validity of PD and IBD diagnoses in the Danish NPR is known to be accurate and almost complete. ${ }^{17} 18$

Potential limitations relate to the registry-based nature of the study, which may imply that atypical parkinsonism syndromes and MSA were registered as idiopathic PD in the ICD-8 disease classification system, where MSA could not be recorded as separate disorder. Register studies may also suffer from detection bias, that is, that diagnosing of one disease may increase the likelihood of concomitant diagnosing of another disease. However, our observation of a nearly constant risk of parkinsonism in all years of follow-up, and hence not limited to the first years after IBD diagnosis, speaks against such initial detection bias. Further, we were unable to access medication data, and considering the higher use of psychotropic medications in persons with IBD than controls we cannot rule out the possibility that some of the increased risk of PD in UC is secondary to drug-induced PD. Finally, information on potential confounders, such as smoking, is not available at the national level.

There is a growing awareness within the scientific communities of the connection between the intestinal environment and the central nervous system (CNS). This so-called 'gut-brain axis' is believed to link functions of the brain with peripheral intestinal functions. ${ }^{2}$ Especially, unbalances in the intestinal microbiome in relation to neurological diseases have recently taken the spotlight, with studies relating PD with a more proinflammatory gut milieu. ${ }^{21}$ Recent evidence for the gut as an initial site of PD pathology comes from a study by Sampson et al demonstrating the importance of the gut microbiota to the pathophysiology of neuroinflammation in the development of motor deficits in the $\alpha$-syn-overexpressing mouse. ${ }^{22}$ In addition, colonisation of these mice by microbiota from patients with PD (relative to microbiota from healthy donors) resulted in worse motor symptoms. ${ }^{22}$ Also, changes in the composition of the gut microbiota have been detected in CD and in UC, reinforcing the hypothesis of dysbiosis as a relevant mechanism underlying IBD pathogenesis. ${ }^{23} 24$

Lewy pathology, resulting from $\alpha$-syn inclusions, the main neuropathological hallmark of PD, can be detected in the GI tract of patients with PD up to 20 years prior to their diagnosis. ${ }^{25}$ However, $\alpha$-syn appears to be abundantly expressed in the ENS in a similar manner in patients with PD, patients with MSA and controls. ${ }^{26}$ Moreover, $p-\alpha-$ syn is detectable in patients with PD and in healthy controls in an age-dependent fashion, questioning the usefulness of $p-\alpha$-syn and native $\alpha$-syn detection in colonic biopsies as a reliable biomarker of PD. ${ }^{62} 28$ Despite this, Barrenschee et al have shown that if a refined morphometric analysis is applied, a specific pattern of $p$ - $\alpha$-syn aggregates can be identified in patients with PD distinct from controls. ${ }^{6}$

The above points raise the question whether the pathological cascade initiates in the CNS or starts in the periphery long before the disease onset. There is a set of non-motor symptoms that may antedate cardinal motor symptoms in PD; these include rapid eye movement sleep behaviour disorder, depression and constipation. ${ }^{29}$ 
The ENS contains a prominent component of glial cells, the so-called enteric glial cells, which, like astrocytes of the CNS, contribute to supporting, protecting and maintaining the neural network. Both IBD and PD have been associated with GI barrier dysfunction and furthermore, the GI symptoms that occur in PD might in part be caused by enteric glial dysfunction. ${ }^{30}$ Recently, it was shown that $\alpha$-syn is a component of the innate immune defence system of the ENS and acts as potent chemoattractant for neutrophils and monocytes, promoting mucosal inflammation. ${ }^{31}$ Inflammation of the GI tract is the signature of IBD. ${ }^{32}$ Notoriously, colonic biopsies from patients with PD show a proinflammatory cytokine expression profiling, for example, interferon gamma (IFN- $\gamma$ ), interleukin (IL)-6 and IL-1 $\beta$, strikingly similar to that reported for IBD. ${ }^{3233} \alpha$-syn may act as an antigenic epitope displayed by major histocompatibility complex inducing the production of proinflammatory cytokines, IL- 5 and IFN- $\gamma$, drive helper and cytotoxic $\mathrm{T}$ cell responses in patients with PD. ${ }^{34}$ These inflammatory processes and intestinal barrier dysfunctions possibly enhance $p$ - $\alpha$-syn assembly into aggregates; however, it remains unclear whether $\alpha$-syn aggregation is a cause or a consequence of inflammation. ${ }^{35}$

Our results suggest a significantly higher risk of PD in patients with IBD compared with non-IBD individuals, and a higher risk in patients with UC than in patients with CD. Interestingly, tobacco smoking is associated with a reduced risk of developing UC (in contrast to $\mathrm{CD})^{36}$ and long-term smoking also reduces the risk of PD. ${ }^{37}$ The biological mechanism behind the protective effects of smoking in UC and PD remains to be clarified, but it is known that both smoking and nicotine affect the composition of the microbiota and reduce the production of proinflammatory cytokines. ${ }^{38}$ The LRRK2 mutation, which may explain between $5 \%$ and $13 \%$ of familial and $1 \%-5 \%$ of sporadic PD, ${ }^{11}$ has been suggested to associate with IBD, especially CD. ${ }^{39}$ Yet, we observed a higher risk of $\mathrm{PD}$ in UC than in patients with CD. The prevalence of the most frequent LRRK2 p.Gly2019S mutation is generally low $(0.55 \%)$ in Danish cohorts of patients with neurodegenerative disorders, even when considering PD cases alone. ${ }^{40}$ This may imply a low involvement of this mutation in the IBD-PD effect in our cohort.

In conclusion, our nationwide population-based cohort study shows a significant association between IBD and later PD. Although the absolute risk of PD remains low, our study points to overlapping pathogenic mechanisms, which merit further investigation, as they may represent targets for future therapeutic interventions.

Acknowledgements We gratefully acknowledge the following foundations for their financial support: Parkinsonforeningen, Landsforeningen Multipel System Atrofi, Aage og Johanne Louis-Hansens Fond and Novo Nordisk Foundation.

Contributors BP, TB, SA and TJ designed the study. TB and MV drafted the first version of the manuscript. All authors contributed substantially to the manuscript. TB and SA did the literature search. MV analysed the data. MV, TJ and TB interpreted the analyses. All authors approved the final manuscript. All authors, external and internal, had full access to all of the data (including statistical reports and tables) in the study and take responsibility for the integrity of the data and the accuracy of the data analysis.

Funding This study was supported by grants from Parkinsonforeningen, Landsforeningen Multipel System Atrofi, and Aage og Johanne Louis-Hansens Fond.

Disclaimer The funding agencies had no role in the design and conduct of the study; the collection, management, analysis and interpretation of the data; the preparation, review or approval of the manuscript; or the decision to submit the manuscript for publication.

Competing interests None declared
Patient consent Not required.

Ethics approval The study was register based and complied with the regulations and registrations set up by the Danish Data Protection Agency and the Danish Health Data Authority (FSEID-00001565).

Provenance and peer review Not commissioned; externally peer reviewed.

Data sharing statement No additional data available.

(C) Article author(s) (or their employer(s) unless otherwise stated in the text of the article) 2019. All rights reserved. No commercial use is permitted unless otherwise expressly granted.

\section{REFERENCES}

1 Khor B, Gardet A, Xavier RJ. Genetics and pathogenesis of inflammatory bowel disease. Nature 2011;474:307-17.

2 Houser MC, Tansey MG. The gut-brain axis: is intestinal inflammation a silent driver of Parkinson's disease pathogenesis? NPJ Parkinsons Dis 2017:3:3:3.

3 Cykowski MD, Coon EA, Powell SZ, et al. Expanding the spectrum of neuronal pathology in multiple system atrophy. Brain 2015;138:2293-309.

4 Spillantini MG, Schmidt ML, Lee VM, et al. Alpha-synuclein in Lewy bodies. Nature 1997;388:839-40.

5 Fujiwara $\mathrm{H}$, Hasegawa M, Dohmae N, et al. Alpha-synuclein is phosphorylated in synucleinopathy lesions. Nat Cell Biol 2002;4:160-4.

6 Barrenschee M, Zorenkov D, Böttner M, et al. Distinct pattern of enteric phosphoalpha-synuclein aggregates and gene expression profiles in patients with Parkinson's disease. Acta Neuropathol Commun 2017;5:1.

7 Sun $\mathrm{M}, \mathrm{He} C$, Cong Y, et al. Regulatory immune cells in regulation of intestinal inflammatory response to microbiota. Mucosal Immunol 2015;8:969-78.

8 Franke A, McGovern DP, Barrett JC, et al. Genome-wide meta-analysis increases to 71 the number of confirmed Crohn's disease susceptibility loci. Nat Genet 2010:42:1118-25.

9 Cookson MR. The role of leucine-rich repeat kinase 2 (LRRK2) in Parkinson's disease. Nat Rev Neurosci 2010;11:791-7.

10 Heckman MG, Schottlaender L, Soto-Ortolaza Al, et al. LRRK2 exonic variants and risk of multiple system atrophy. Neurology 2014;83:2256-61.

11 Kumari U, Tan EK. LRRK2 in Parkinson's disease: genetic and clinical studies from patients. Febs J 2009;276:6455-63.

12 Moehle MS, Webber PJ, Tse T, et al. LRRK2 inhibition attenuates microglial inflammatory responses. J Neurosci 2012;32:1602-11.

13 Brudek T, Winge K, Folke J, et al. Autoimmune antibody decline in Parkinson's disease and Multiple System Atrophy; a step towards immunotherapeutic strategies. Mol Neurodegener 2017;12:44.

14 Lin JC, Lin CS, Hsu CW, et al. Association Between Parkinson's Disease and Inflammatory Bowel Disease: a Nationwide Taiwanese Retrospective Cohort Study. Inflamm Bowel Dis 2016:22:1049.

15 Pedersen CB. The Danish Civil Registration System. Scand J Public Health 2011:39:22-5.

16 Lynge E, Sandegaard JL, Rebolj M. The Danish National Patient Register. Scand J Public Health 2011;39:30-3.

17 Fonager K, Sørensen HT, Rasmussen SN, et al. Assessment of the Diagnoses of Crohn's Disease and Ulcerative Colitis in a Danish Hospital Information System. Scand J Gastroenterol 1996;31:154-9.

18 Wermuth L, Lassen CF, Himmerslev L, et al. Validation of hospital register-based diagnosis of Parkinson's disease. Dan Med J 2012;59:A4391.

19 Wermuth L, Cui X, Greene N, et al. Medical Record Review to Differentiate between Idiopathic Parkinson's Disease and Parkinsonism: A Danish Record Linkage Study with 10 Years of Follow-Up. Parkinsons Dis 2015;2015:781479.

20 Christensen S, Johansen MB, Christiansen CF, et al. Comparison of Charlson comorbidity index with SAPS and APACHE scores for prediction of mortality following intensive care. Clin Epidemiol 2011;3:203-11.

21 Tremlett H, Bauer KC, Appel-Cresswell S, et al. The gut microbiome in human neurological disease: a review. Ann Neurol 2017;81:369-82.

22 Sampson TR, Debelius JW, Thron T, et al. Gut Microbiota Regulate Motor Deficits and Neuroinflammation in a Model of Parkinson's Disease. Cell 2016;167:1469-80.

23 Halfvarson J, Brislawn C, Lamendella R, et al. Dynamics of the human gut microbiome in inflammatory bowel disease. Nat Microbiol 2017.

24 Pascal V, Pozuelo M, Borruel N, et al. A microbial signature for Crohn's disease. Gut 2017;66:813-22.

25 Stokholm MG, Danielsen EH, Hamilton-Dutoit SJ, et al. Pathological $\alpha$-synuclein in gastrointestinal tissues from prodromal Parkinson disease patients. Ann Neurol 2016;79:940-9.

26 Chung S, Kim J, Lee $\mathrm{H}$, et al. Alpha-synuclein in gastric and colonic mucosa in Parkinson's disease: Limited role as a biomarker. Movement Disorders 2016;31:241-9.

27 Schneider SA, Boettner M, Alexoudi A, et al. Can we use peripheral tissue biopsies to diagnose Parkinson's disease? A review of the literature. Eur J Neurol 2016;23:247-61 
28 Böttner M, Zorenkov D, Hellwig I, et al. Expression pattern and localization of alpha-synuclein in the human enteric nervous system. Neurobiol Dis 2012;48:474-80.

29 Schapira AHV, Chaudhuri KR, Jenner P. Non-motor features of Parkinson disease. Nat Rev Neurosci 2017;18:435-50.

30 Clairembault T, Leclair-Visonneau L, Neunlist M, et al. Enteric glial cells: New players in Parkinson's disease? Movement Disorders 2015;30:494-8.

31 Stolzenberg E, Berry D, Yang D, et al. A Role for Neuronal Alpha-Synuclein in Gastrointestinal Immunity. J Innate Immun 2017.

32 Devos D, Lebouvier T, Lardeux B, et al. Colonic inflammation in Parkinson's disease. Neurobiol Dis 2013;50:42-8.

33 Neurath MF. Cytokines in inflammatory bowel disease. Nat Rev Immunol 2014;14:329-42.

34 Sulzer D, Alcalay RN, Garretti F, et al. Erratum: T cells from patients with Parkinson's disease recognize $\alpha$-synuclein peptides. Nature 2017;549:292.
35 Gao H, Zhang F, Zhou H, et al. Neuroinflammation and Alpha-Synuclein Dysfunction Potentiate Each Other, Driving Chronic Progression of Neurodegeneration in a Mouse Model of Parkinsons Disease. Environ Health Perspect.

36 To N, Ford AC, Gracie DJ. Systematic review with meta-analysis: the effect of tobacco smoking on the natural history of ulcerative colitis. Aliment Pharmacol Ther 2016:44:117-26.

37 Li X, Li W, Liu G, et al. Association between cigarette smoking and Parkinson's disease: A meta-analysis. Arch Gerontol Geriatr 2015;61:510-6.

38 Derkinderen P, Shannon KM, Brundin P. Gut feelings about smoking and coffee in Parkinson's disease. Movement Disorders 2014;29:976-9.

39 Witoelar A, Jansen IE, Wang Y, et al. Genome-wide Pleiotropy Between Parkinson Disease and Autoimmune Diseases. JAMA Neurol 2017;74:780-792.

40 Bech S, Nørremølle A, Winge K, et al. The Irrk2 p.Gly2019Ser mutation is uncommon in a Danish cohort with various neurodegenerative disorders. Parkinsonism Relat Disord 2011;17:398-9. 